\title{
Is there a non-monotonic relation between photospheric brightness and magnetic field strength in solar plage regions?
}

\author{
D. Röhrbein ${ }^{1,2}$, R. Cameron ${ }^{1}$, and M. Schüssler ${ }^{1}$
}

\author{
${ }^{1}$ Max-Planck-Institut für Sonnensystemforschung, Max-Planck-Str. 2, 37191 Katlenburg-Lindau, Germany \\ e-mail: roehrbein@mps .mpg . de \\ 2 Technische Universität Braunschweig, Institut für Theoretische Physik, Mendelssohnstrasse 3, 38106 Braunschweig, Germany
}

Received 15 April 2011 / Accepted 6 July 2011

\begin{abstract}
Context. The relationship between the brightness and field strength of small-scale solar magnetic features is an important factor for solar irradiance variations and a constraint for simulations of solar magneto-convection.

Aims. We wish to clarify the origin of the apparent discrepancy between observational results and radiative MHD simulations.

Methods. Maps of (bolometric) brightness and magnetic field strength from the simulation of a plage region were convolved and rebinned to mimic observations obtained with telescopes with finite aperture.

Results. Image smearing changes the monotonic relation between brightness and field strength obtained at the original resolution of the simulation into a profile with a maximum at intermediate field strength, which is in qualitative agreement with the observations. This result is mainly due to the smearing of strong magnetic fields at the bright edges of magnetic structures into the weakly magnetized adjacent areas.

Conclusions. Observational and simulation results are qualitatively consistent with each other if the finite spatial resolution of the observations is taken into account.
\end{abstract}

Key words. Sun: surface magnetism - Sun: photosphere

\section{Introduction}

Solar photospheric bright points visible in the continuum and in various spectral bands are indicators of magnetic flux concentrations (Zwaan 1978; Solanki 1993; Berger \& Title 2001; Ishikawa et al. 2007; de Wijn et al. 2009). They are thought to form through the evacuation of magnetic flux concentrations in the $\mathrm{kG}$ regime (Spruit 1976; Parker 1978) in a state of total pressure balance, $p_{\mathrm{e}}=p_{\mathrm{i}}+B^{2} / 8 \pi$, where $p_{\mathrm{e}}$ and $p_{\mathrm{i}}$ are the external and internal pressures, respectively, and $B$ is the field strength. The resulting local depression of the surface of optical depth unity leads to lateral radiative heating from the hot walls of the flux concentration. This heating dominates the magnetic suppression of convective heat transport for small flux concentrations, leading to bright features. Larger flux concentrations, such as pores and sunspots, are dark since the heating by the hot wall affects only a boundary region that corresponds to optical depth unity in the horizontal direction.

From these considerations one expects a monotonic relation between the brightness and field strength of small-scale magnetic features (i.e., those small enough to be efficiently heated by the hot walls): for increasing field strength, the decreasing internal gas pressure leads to deeper levels of the surface of optical depth unity, which entails an increasing brightness. In fact, such a monotonic average relation is found in numerical 3D radiative MHD simulations (Vögler et al. 2005, see their Fig. 13), although the variable environment of the magnetic features leads to considerable scatter. On the other hand, observations (Title et al. 1989; Topka et al. 1992; Title et al. 1992; Lawrence et al. 1993; Stangl \& Hirzberger 2005; Narayan \& Scharmer 2010; Schnerr \& Spruit 2011) typically show a different relation between magnetic field signal and contrast (relative difference to the average disk-center brightness). Either the contrast is found to be monotonically decreasing with increasing field (possibly with a "knee" at intermediate values) or the relation is not monotonic: after initially increasing for weak fields, the contrast reaches a maximum and then decreases again for stronger fields, even if pores are explicitly removed from the analysis (Kobel et al. 2011).

It is well known that spatial smearing can significantly affect the contrast and magnetic signature of small-scale features (e.g., Title \& Berger 1996). Owing to the effects of finite spatial resolution, seeing, and straylight, observations typically do not provide the actual field strength but some kind of average over the resolution element and the range of straylight. This quantity is sometimes called "flux density" in order to distinguish it from the (generally unknown) actual magnetic field strength within a magnetic feature. Therefore, one cannot necessarily expect the simulated and observed mean relationships to coincide since they refer to different quantities. The aim of this paper is to evaluate the effect of finite spatial resolution on the comparison of observational and simulation results. For a first qualitative assessment of the effect, we simply convolve bolometric intensity and magnetic field maps from simulations with Airy functions that correspond to telescope apertures of $1.0 \mathrm{~m}$ and $0.5 \mathrm{~m}$, respectively, and rebin to the corresponding pixel size for critical sampling at the given spatial resolution. We consider the effect of this spatial smearing on the average pixel-by-pixel relation between intensity and magnetic field. This simple approach ignores important observational effects such as noise, straylight, and telescope obscurations, and thus is not intended for a quantitative comparison with observations. This would also involve 
Table 1. Pixel size values for the three resolutions considered.

\begin{tabular}{lccc}
\hline \hline Resolution & \# Pixels & $\mathrm{km} / \mathrm{px}$ & $\mathrm{arcsec} / \mathrm{px}$ \\
\hline MURaM full & $1152 \times 1152$ & 20.83 & 0.028 \\
$1.0 \mathrm{~m}$ & $414 \times 414$ & 58 & 0.08 \\
$0.5 \mathrm{~m}$ & $207 \times 207$ & 116 & 0.16 \\
\hline
\end{tabular}

determining the magnetic field from synthetic Stokes profiles, which is beyond the scope of this paper. Here, we wish to study the fundamental effects of image smearing, deferring a more detailed comparison with observations to a subsequent study.

The relevance of a proper understanding of the relation between brightness and magnetic field goes far beyond its role as a constraint for simulations because the brightness of smallscale magnetic features is a crucial factor for the variations in total solar irradiance over timescales between days and decades (Krivova et al. 2003, 2006; Domingo et al. 2009).

\section{MHD simulation}

We used the MURaM code (Vögler 2003; Vögler et al. 2005) with essentially the same setup as in Vögler et al. (2005). The simulation was performed to represent a plage region with a mean vertical magnetic field of $200 \mathrm{G}$ inside the computational domain for a duration of $3 \mathrm{~h}$ solar time. The dimensions of the domain were $1400 \mathrm{~km}$ in the vertical and $24 \mathrm{Mm} \times 24 \mathrm{Mm}$ in the horizontal directions with a mesh of $100 \times 1152 \times 1152$ grid points. This corresponds to a $32^{\prime \prime} \times 32^{\prime \prime}$ horizontal field of view on the Sun. In the vertical direction, the simulation covers the upper layers of the convection zone and the photospheric layers, with the mean level of the (Rosseland) optical depth $\tau_{\mathrm{R}}=1$ being located about $400 \mathrm{~km}$ below the upper boundary.

\section{Convolution for different telescope apertures}

We consider maps of the vertically emerging bolometric intensity $(I)$ and the vertical component of the magnetic field $\left(B_{\text {vert }}\right)$ on the surface $\tau_{\mathrm{R}}=1$ from one snapshot of the simulation. Owing to the large size of the computational domain, this was found to be sufficient for obtaining proper statistics. The $I$ and $B_{\text {vert }}$ maps were convolved with an Airy function that corresponds to telescope apertures of $D=1.0 \mathrm{~m}$ and $D=0.5 \mathrm{~m}$, respectively, and a wavelength of $\lambda=630.2 \mathrm{~nm}$. After convolution, these images were rebinned to a pixel size of $\Delta x / 2$, corresponding to critical sampling according to the Rayleigh limit, $\Delta x=1.22 \lambda / D$. Table 1 gives the values of the pixel resolution for the original and convolved cases.

Figure 1 illustrates the effect of the smearing on the visibility of simulated small-scale features. For clarity, we only show a $6 \mathrm{Mm} \times 6 \mathrm{Mm}$ section of the full map, corresponding to $1 / 16$ of the actual snapshot size. The subsequent analysis was carried out using the full $24 \mathrm{Mm} \times 24 \mathrm{Mm}$ maps.

\section{Results}

For our analysis, we consider the intensity contrast, $C=I /\langle I\rangle-$ 1 , and the corresponding vertical magnetic field $B_{\mathrm{vert}}$ at $\tau_{\mathrm{R}}=1$ for each pixel. Here, $\langle I\rangle$ represents the mean bolometric intensity of the whole snapshot. There are no pores in this simulation because the largest magnetic features are micropores with essentially neutral or moderately negative average contrast. Figure 2 shows contours of a 2D histogram giving the number of pixels as a smoothed function of the intensity contrast and the vertical magnetic field strength. The histogram is consistent with Fig. 13 of Vögler et al. (2005).

Figure 3 shows the average contrast for $B_{\text {vert }}$ bins containing 1000 pixels each at original resolution, and 200 pixels each for $1.0 \mathrm{~m}$ aperture and for $0.5 \mathrm{~m}$ aperture. The basic features in Fig. 3 include that: (1) all curves show a peak with positive contrast for weak field $\left(\left|B_{\text {vert }}\right| \lesssim 100 \mathrm{G}\right)$. These pixels mainly correspond to weakly magnetized regions within granules and are essentially unaffected by the resolution; (2) for $\left|B_{\text {vert }}\right|$ between $\sim 100 \mathrm{G}$ and $300 \mathrm{G}$, the mean intensity contrast decreases with increasing magnetic field, because the magnetic flux is advected and concentrated by flux expulsion into the dark intergranular lanes and because the field is too weak to have a significant effect on the contrast. At original MURaM resolution, the mean contrast for $\left|B_{\text {vert }}\right| \gtrsim 300 \mathrm{G}$ increases monotonically with increasing field strength because the flux tubes become increasingly evacuated and brighten (Spruit 1976). The average contrast reaches positive values up to nearly $30 \%$ for $B_{\text {vert }} \gtrsim 1500 \mathrm{G}$.

For $1.0 \mathrm{~m}$ telescope aperture, there is an increase in the mean contrast for $B_{\text {vert }} \gtrsim 200 \mathrm{G}$, but it saturates at around $1200 \mathrm{G}$. The maximum mean contrast reached at $B_{\text {vert }} \approx 1200 \mathrm{G}$ is about $3 \%$. For $0.5 \mathrm{~m}$ telescope aperture, the mean contrast reaches a maximum at around $1000 \mathrm{G}$ and then decreases again, so that at this resolution strong magnetic features appear up to $10 \%$ darker than the mean intensity. In summary, while the mean contrast curve at original simulation resolution confirms the expectations outlined in the introduction (in the absence of pores), for reduced resolution it saturates or even turns over for strong fields, which is in accordance with observational results at similar spatial resolution (Narayan \& Scharmer 2010; Kobel et al. 2011; Schnerr $\&$ Spruit 2011). The contrast peak occurs at a somewhat higher field than in the observations. This is probably because we take the simulation values at the $\tau_{\mathrm{R}}=1$ surface, while observations typically sample $0.01 \lesssim \tau_{\mathrm{R}} \lesssim 0.1$. A quantitative comparison with observations would require determining $B$ from (spatially smeared) synthetic Stokes profiles. Such an analysis is beyond the scope of this paper.

How can we explain the saturation and turnover of the contrast curve for smeared images? We first checked whether the rebinning to critical sampling is the reason, but we essentially found the same results in the case without rebinning. Next, we separately considered the effect of smearing the intensity and smearing the magnetic field. The upper panel of Fig. 4 shows the mean contrast curve resulting from convolving only the intensity image but keeping the original $B_{\text {vert }}$ map, whereas the lower panel shows the reverse case: original intensity and convolved $B_{\text {vert }}$. No spatial rebinning was applied, and $B_{\text {vert }}$ was binned with 1000 pixels per bin in both cases.

Evidently, the smearing of the intensity alone results in an overall reduction in contrast, but does not lead to the saturation and the turnover seen in Fig. 3. In contrast, the smearing of $B_{\text {vert }}$ alone clearly leads to a turnover at reduced resolution. For intermediate values of $B_{\text {vert }}$, there is a positive contrast up to $5 \%$, whereas for stronger magnetic field, we find a negative contrast of up to $-10 \%$.

That smearing the intensity alone does not lead to an overturn of the mean contrast curve provides the essential clue for understanding the effect of finite resolution. The magnetic features in the simulated plage region represent a mixture of small intergranular bright flux concentrations and somewhat bigger micropores with mostly neutral or negative contrast, except at their bright edges. When the intensity alone is smeared, the association of strong field with positive contrast of many 
D. Röhrbein et al.: Relation between brightness and magnetic field
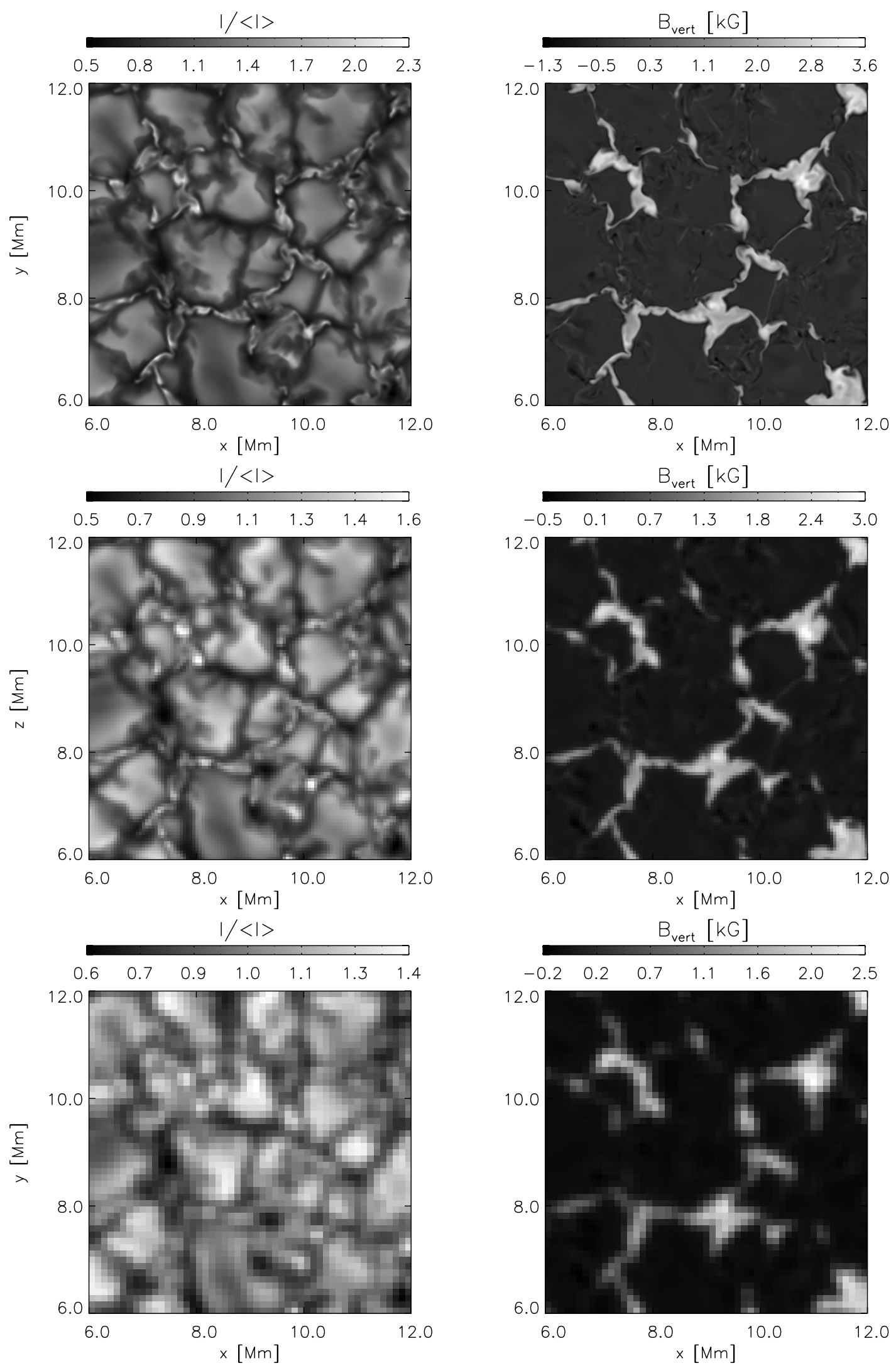

Fig. 1. Effect of convolution and rebinning on maps of bolometric intensity (left panels) and vertical field strength on the $\tau_{\mathrm{R}}=1$ surface $($ right panels). Top row: original simulation resolution; middle row: convolved and rebinned corresponding to $1.0 \mathrm{~m}$ telescope apertures; bottom row: convolved and rebinned corresponding to $0.5 \mathrm{~m}$ telescope aperture. For clarity, we show only a $6 \mathrm{Mm} \times 6 \mathrm{Mm}$ section of the full $24 \mathrm{Mm} \times 24 \mathrm{Mm}$ full horizontal areas of the simulation. 


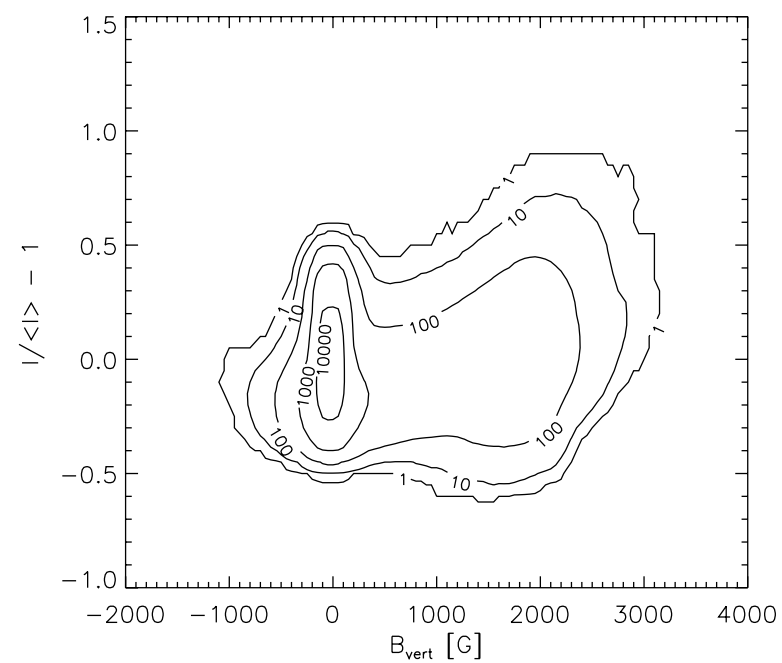

Fig. 2. Contour plot of the $2 \mathrm{D}$ histogram of the number of pixels at original resolution in bins of 5\% contrast and $50 \mathrm{G}$ vertical field. Pixel numbers for contrast over $20 \%$ and field strength over $200 \mathrm{G}$ were smoothed.

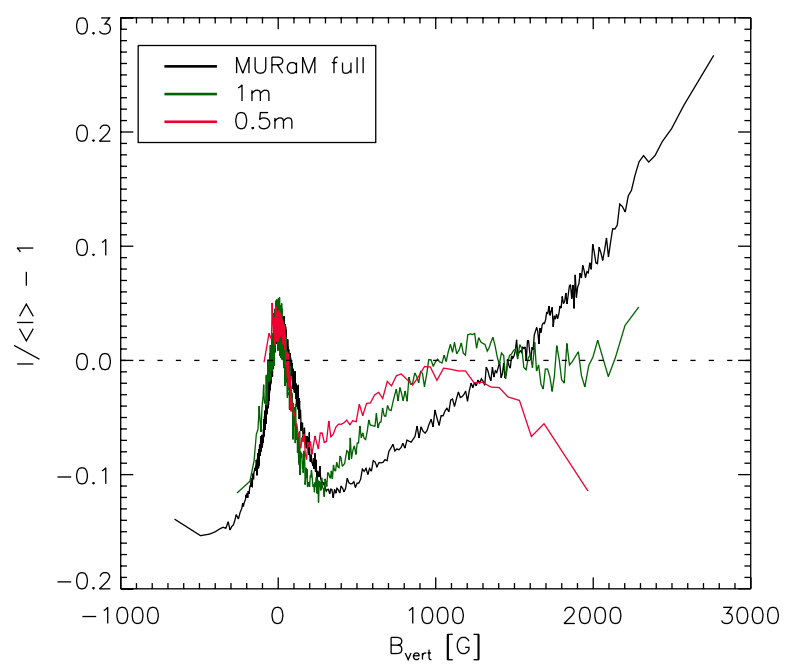

Fig. 3. Bin-averaged intensity contrast vs. $B_{\text {vert }}$ at original resolution (black), for the convolved intensity and magnetic field maps for $1.0 \mathrm{~m}$ telescope aperture (green), and for $0.5 \mathrm{~m}$ telescope aperture (red).

small-scale features remains unaffected, although their peak brightness is somewhat reduced. The micropores are less affected by smearing. As a result, the mean contrast curve is shifted downward for high field values, but does not turn over. However, if we smear the magnetic field, then the field values in the small bright features are strongly reduced (since surrounded by almost zero field), and the corresponding points in the $I-B$ diagram are shifted leftward to lower field values. This does not happen in the bulk of the bigger micropores, so that the points representing them remain in the strong-field bins. Upon averaging, this leads to a downturn of the contrast curve for strong fields and to higher mean intensity levels at intermediate fields, since this is the region into which the bright strong-field pixels are shifted. In summary, this means that the downturn of the contrast curve results from the "loss" of bright strong-field pixels in the small flux concentrations, while the darker strong-field pixels in the micropores largely remain.

The effect of smearing the magnetic field described above is illustrated in Fig. 5. Regions with a strong vertical magnetic field, $B_{\text {vert }}>1500 \mathrm{G}$, at original resolution are outlined in yellow
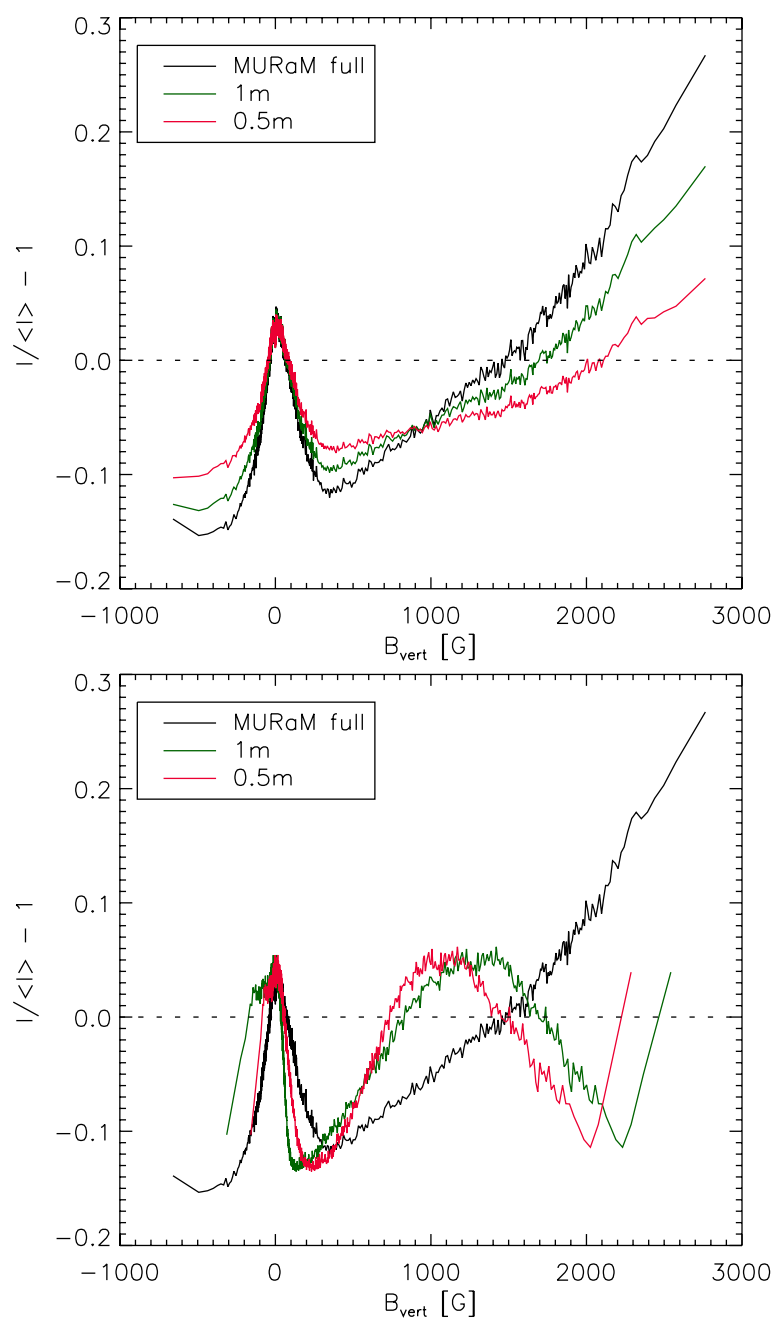

Fig. 4. Same as in Fig. 3, but after convolving only the intensity image (upper panel) and after convolving only the magnetic field map (lower panel). The maps have not been rebinned in either case.

on top of a normalized intensity map, in comparison to the regions with $B_{\text {vert }}>1500 \mathrm{G}$ at $0.5 \mathrm{~m}$ telescope aperture (outlined in red). We chose the threshold at $1500 \mathrm{G}$, because the mean contrast at original resolution becomes positive at this value. The figure shows that in the transition from original resolution to $0.5 \mathrm{~m}$ telescope aperture we lose most of the small bright features of high field strength, mainly because their field becomes smeared with adjacent regions of low field strength. This applies both for the thin sheet-like features between granules and for the bright edges of micropores (e.g., the feature centered at $x=2.3 \mathrm{Mm}, y=2 \mathrm{Mm}$ in the bottom panel of Fig. 5). What appears as a strong field after smearing (see Fig. 1, lower panel) mainly corresponds to the central regions of the micropores, which tend to be darker, because the lateral heating diminishes with increasing distance from the hot wall (Spruit 1976).

Figure 6 illustrates the shift of pixels in the $I-B$ diagram due to smearing, which leads to the turnover of the average contrast curve. The plot in the upper panel shows that the smearing most strongly affects the pixels on the upper righthand side of the histogram, i.e., those with high contrast and high field strength. The plot in the lower panel is derived from the histogram at original resolution in the upper panel, but now the pixels are divided into those with $B_{\text {vert }}>1500 \mathrm{G}$ after convolution to $0.5 \mathrm{~m}$ telescope aperture and all other pixels. The region with $B_{\text {vert }}>1500 \mathrm{G}$, 

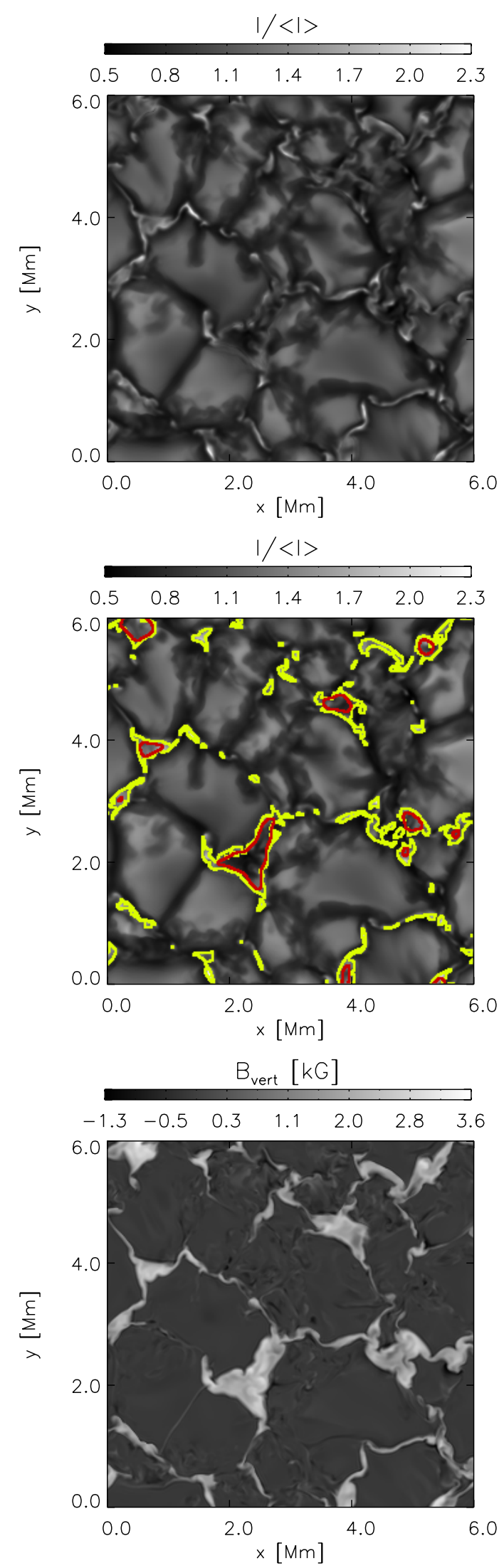

Fig. 5. Normalized continuum intensity (upper panel) and the corresponding vertical magnetic field (lower panel) at original resolution. The middle panel shows the same intensity image as the top panel, but with regions of $B_{\text {vert }}>1500 \mathrm{G}$ outlined in yellow for original resolution and outlined in red for the resolution corresponding to $0.5 \mathrm{~m}$ telescope aperture.
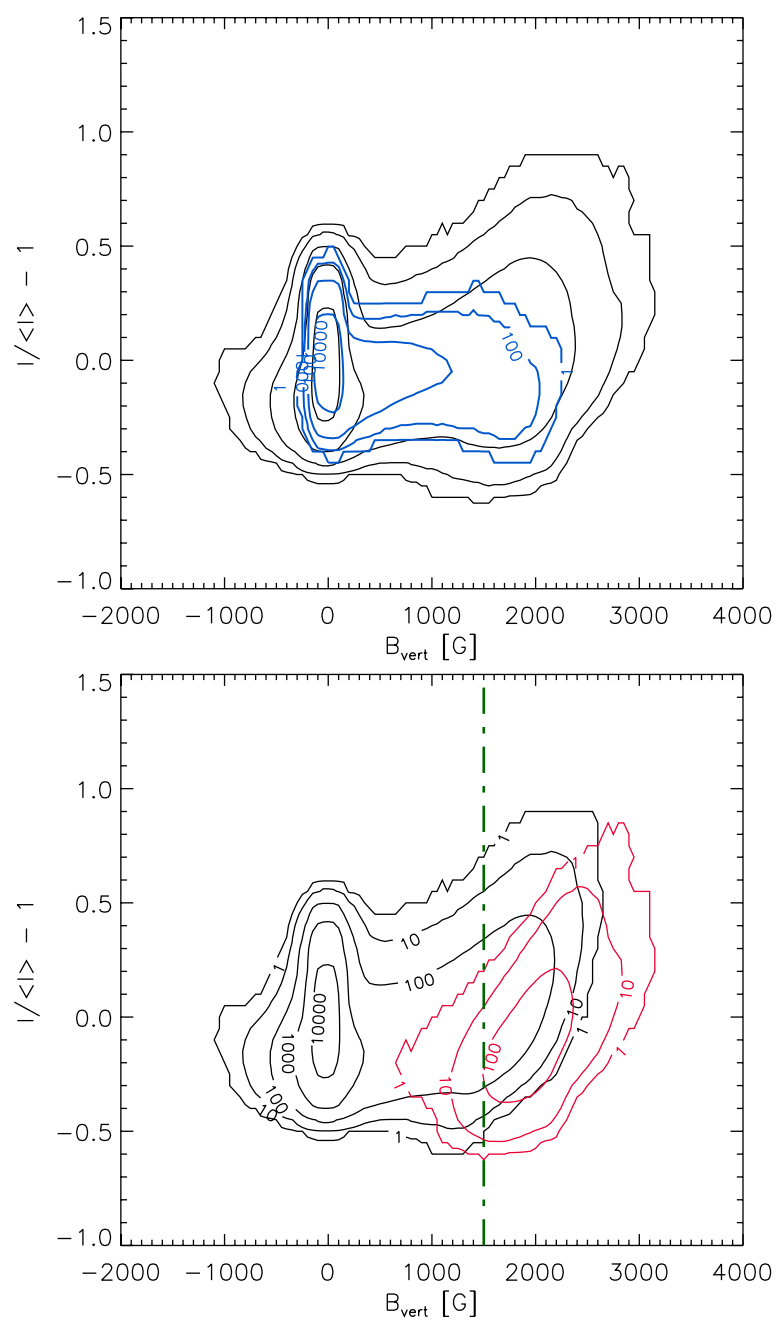

Fig. 6. Upper panel: contour plot of the $2 \mathrm{D}$ histogram of the number of pixels at original resolution, binned and smoothed (black lines). The contours and labels for the original distribution are the same as in Fig. 2. Overlaid are the contours for the case after convolution and rebinning, corresponding to $0.5 \mathrm{~m}$ aperture, also binned and smoothed (blue contours). Lower panel: contour plot of the $2 \mathrm{D}$ histogram of the number of pixels at original resolution, with pixels divided into two groups (red \& black contours): pixels with $B_{\text {vert }}>1500 \mathrm{G}$ after convolution to $0.5 \mathrm{~m}$ telescope aperture are shown with red contours. These correspond to the pixels outlined in red in the middle panel of Fig. 5. The remaining pixels correspond to the black contours. The vertical dash-dotted line indicates the field strength of $1500 \mathrm{G}$ chosen for the separation.

where the red and the black distributions do not overlap contains the bright pixels from the edges of micropores and the thin, elongated intergranular magnetic features at original resolution that are transferred into bins with low and intermediate field in the smeared maps. This leads to the shift, saturation, and overturn of the mean contrast curves.

In the lower left of the red contours in the upper panel of Fig. 6, we find pixels that actually increase their field values by smearing. At original resolution, these pixels typically show intermediate field strength and neutral or negative contrast. Most of them correspond to regions at the periphery of flux concentrations or patches of lower field in micropores, so that the field values increase by smearing with nearby strong-field pixels.

The mechanism for overturning the contrast curve described above relies on the existence of a population of micopores with neutral or negative intensity contrast and a strong field, which 
are less affected by smoothing than the bright small-scale intergranular magnetic features. In fact, a simulation with much less net vertical magnetic flux (mean vertical field of $10 \mathrm{G}$ ) does not show the overturning after smoothing. On the other hand, Kobel et al. (2011) also find an overturning contrast curve for what they call a region of "quiet Sun network". In line with our interpretation of the overturn in terms of a finite-resolution effect, we would then suggest that this observed region contained a considerable amount of flux in the form of micropores. As a further observational test, it would be interesting to consider the mean contrast curve of a pure internetwork region without micropores.

\section{Discussion and conclusion}

We have shown that the simulated and observational relations between intensity contrast and magnetic field in plage regions become qualitatively consistent if the spatial smearing due to finite observational resolution is taken into account. In particular, the downturn of the observed mean contrast curve for strong fields can be understood mainly as an artifact resulting from the smearing of the magnetic field. Without this smearing, the contrast curve is monotonic and shows the highest contrast for the strongest fields, as long as proper pores are excluded.

In subsequent work, we will provide a quantitative comparison with observational results, deriving the magnetic field from synthetic Stokes profiles and including further instrumental effects.
Acknowledgements. D.R. acknowledges a Ph.D. fellowship from the International Max Planck Research School on Physical Processes in the Solar System and Beyond. We would like to thank the referee for the useful corrections.

\section{References}

Berger, T. E., \& Title, A. M. 2001, ApJ, 553, 449

de Wijn, A. G., Stenflo, J. O., Solanki, S. K., \& Tsuneta, S. 2009, Space Sci. Rev., 144,275

Domingo, V., Ermolli, I., Fox, P., et al. 2009, Space Sci. Rev., 145, 337

Ishikawa, R., Tsuneta, S., Kitakoshi, Y., et al. 2007, A\&A, 472, 911

Kobel, P., Solanki, S. K., \& Borrero, J. M. 2011, A\&A, 531, A112

Krivova, N. A., Solanki, S. K., Fligge, M., \& Unruh, Y. C. 2003, A\&A, 399, L1

Krivova, N. A., Solanki, S. K., \& Floyd, L. 2006, A\&A, 452, 631

Lawrence, J. K., Topka, K. P., \& Jones, H. P. 1993, J. Geophys. Res., 98, 18911

Narayan, G., \& Scharmer, G. B. 2010, A\&A, 524, A3

Parker, E. N. 1978, ApJ, 221, 368

Schnerr, R. S., \& Spruit, H. C. 2011, A\&A, 532, A136

Solanki, S. K. 1993, Space Sci. Rev., 63, 1

Spruit, H. C. 1976, Sol. Phys., 50, 269

Stangl, S., \& Hirzberger, J. 2005, A\&A, 432, 319

Title, A. M., \& Berger, T. E. 1996, ApJ, 463, 797

Title, A. M., Tarbell, T. D., Topka, K. P., et al. 1989, ApJ, 336, 475

Title, A. M., Topka, K. P., Tarbell, T. D., et al. 1992, ApJ, 393, 782

Topka, K. P., Tarbell, T. D., \& Title, A. M. 1992, ApJ, 396, 351

Vögler, A. 2003, Ph.D. Thesis, University of Göttingen, Germany http://webdoc. sub.gwdg.de/diss/2004/voegler

Vögler, A., Shelyag, S., Schüssler, M., et al. 2005, A\&A, 429, 335

Zwaan, C. 1978, Sol. Phys., 60, 213 